\title{
Per una storia di Il sorriso dell'ignoto marinaio di Vincenzo Consolo
}

\author{
Nicolò Messina
}

Universitat de Girona

\section{Abstract}

Il contributo tenta di delineare la storia del farsi dell'opera piú studiata di Vincenzo Consolo sulla scorta dei testimoni già sottoposti a recensio (edizioni a stampa, dattiloscritti, manoscritti).

Parole chiave: Consolo, Sorriso dell'ignoto marinaio, edizioni critico-genetiche, ecdotica di testi moderni e contemporanei.

\section{Abstract}

The contribution attempts to outline the history of the creation of Vincenzo Consolo's most studied work, based on the supply of accounts already submitted to review (printed, typed and manuscript editions).

Key words: Consolo, Sorriso dell'ignoto marinaio, critical-genetic editions, critical editions of modern and contemporaneous texts.

\section{In limine}

Nella presentazione della nuova collana «Clásicos Modernos» di una delle piú prestigiose case editrici spagnole, José Saramago, senz'altro nel suo castigliano deliziosamente lusitaneggiante e con il suo abituale tono deciso, asseriva pubblicamente: «Estamos hechos de pasado. El presente no existe y el futuro no sabemos lo que es». ${ }^{1}$ La frase potrebbe ben costituire l'esergo di queste pagine, che hanno per oggetto-soggetto Il sorriso dell'ignoto marinaio, e l'aggancio è almeno doppio.

1. L'incontro pubblico, organizzato dalle edizioni Alfaguara, si è tenuto al Círculo de Bellas Artes di Madrid il 27 settembre 2004. L'idea della collana è dovuta — a detta della stessa direttrice editoriale di Alfaguara, Amaya Elezcano - a un suggerimento del Nobel portoghese. La collana, inaugurata da Jacques el fatalista di Denis Diderot, annovera tra i primi volumi, già in libreria, anche i manzoniani Los novios nella traduzione fattane da Esther Benítez. Cfr. El País (Martes 28 de septiembre de 2004): 42. 
Da un lato, infatti, e non appaia aneddotico, il Sorriso è stato la scorsa primavera ripubblicato da Mondadori in una collana che curiosamente ricorre alla medesima etichetta: "Classici moderni»; ${ }^{2}$ dall'altro, poi, l'affondo di Saramago — non a sproposito in un oggi affetto da multiformi amnesie — rivendica in sé e per sé il ruolo della memoria senza la quale non siamo, e non certo perché atteggiati a conservatori idolatri del vissuto umano, perché abbarbicate, irremovibili ostriche verghiane ${ }^{3}$ o stanchi e immalinconiti laudatores temporis acti. $\mathrm{Al}$ riguardo, quale migliore sintonia con Consolo? Il quale - è risaputo — da sempre s'oppone vigile all'appiattimento stritolante sull'unica dimensione temporale del presente, comodo, se non programmaticamente ricercato dagli autarchi che s'ispirano al pensiero unico. Ecco perché forse Consolo, da sempre, fa letteratura ricorrendo a metafore storiche. D'altra parte, come piú di uno ha sottolineato, è certo intorno alla funzione attiva, alla forza propulsiva della memoria che quaglia la metafora del Sorriso: un ieri, ottocentescamente databile, in dialettica con l'oggi del lettore (la metà degli anni Settanta del secolo breve appena concluso, ma anche la metà del primo decennio di questo nostro nuovo secolo). ${ }^{4}$

2. Cfr. piú avanti il riferimento bibliografico completo.

3. Per fugare ogni possibile dubbio sulla propulsività della memoria, da non intendere pertanto quale attaccamento [...] allo scoglio di un rassegnato immobilismo, non è fuori luogo citare per esteso, il noto passo di Fantasticheria (1879), che, pur estrapolato dal suo contesto e con tutti i sottili distinguo dell'autore, sembra presago di un certo fatalismo misoneista, improntato piú all'inutilità che all'impossibilità di ogni reazione umana alle condizioni e ai ruoli assegnati; manifestazione, in breve, di una sorta di noluntas: "Insomma l'ideale dell'ostrica! direte voi. - Proprio l'ideale dell'ostrica, e noi non abbiamo altro motivo di trovarlo ridicolo che quello di non esser nati ostriche anche noi. Per altro il tenace attaccamento di quella povera gente allo scoglio sul quale la fortuna li ha lasciati cadere mentre seminava príncipi di qua e duchesse di là, questa rassegnazione coraggiosa ad una vita di stenti, questa religione della famiglia, che si riverbera sul mestiere, sulla casa, e sui sassi che la circondano, mi sembrano - forse pel quarto d'ora - cose serissime e rispettabilissime anch'esse. Parmi che le irrequietudini del pensiero vagabondo s'addormenterebbero dolcemente nella pace serena di quei sentimenti miti, semplici, che si succedono calmi e inalterati di generazione in generazione.» (G. VERGA, Tutte le novelle, ed. Carla RICCARDI, Milano: Mondadori, $1979 ; 1996^{5}$, p. $\left.135-136\right)$

4. Per felici coincidenze, alle giornate sivigliane all'origine di queste note partecipava anche Maria Attanasio, fine autrice di poesie (Interni, Parma: Guanda, 1979; Nero barocco nero, Caltanissetta-Roma: Sciascia, 1985; Eros e mente, Milano: La Vita Felice, 1996; Amnesia del movimento delle nuvole, Milano: La Vita Felice, 2003; 2004 ${ }^{3}$ ), che si rivela scossa dall'identica volontà di resistenza all'oblio, a giudicare dai frutti delle ore da lei dedicate alla prosa: Correva l'anno 1698 e nella città avvenne il fatto memorabile, Palermo: Sellerio, 1994; Piccole cronache di un secolo, Palermo: Sellerio, 1997 (con Domenico AMOroso); Di Concetta e le sue donne, Palermo: Sellerio, 1999. Proprio da quest'ultimo, commosso, bel librotestimonianza si estrapolano deliberatamente due brani assai eloquenti sull'etica dello scrivere: «Un tempo ogni città, piccola o grande, affidava la storia civile della comunità alla scrittura del cronista; insieme agli eventi civici e allo straordinario egli spesso registrava anche l'ordinario di essa [...] sottraendone la memoria alle azzeranti generalizzazioni della storia, che per sua natura emargina in un'impenetrabile zona d'ombra l'alfa e l'omega costitutivi della sua trama» (p. 32); «Non restava che [...] testimoniare direttamente questa piccola storia di ordinaria militanza, una tra le tante di quegli anni. || Senza però sottrarsi al 


\section{De finibus terminisque constituendis}

$\mathrm{Su}$ Consolo e Il sorriso dell'ignoto marinaio, in particolare, l'interesse critico non è mai tramontato. Ingente è ormai la letteratura secondaria. Da un lato, ne fanno fede le bibliografie via via aggiornate e desumibili da volumi e riviste: dalla prima monografia di Flora Di Legami ${ }^{5}$ al numero omaggio di Nuove Effemeridi, ${ }^{6}$ dal libro di Attilio Scuderi ${ }^{7}$ a quello recente di Giuseppe Traina, ${ }^{8}$ dal "ritratto» di Enzo Papa ${ }^{9}$ alla premessa editoriale dell'ultima ristampa dell'opera. ${ }^{10}$ Dall'altro, chiara eco se ne riceve anche da collectanea a seguito di convegni dedicati allo scrittore: si rammentino almeno quelli organizzati nel solo ultimo torno di tempo a Parigi, Siracusa, Siviglia. ${ }^{11}$

Anche i lettori, dilettanti e non solo professionisti della letteratura, compresi quanti si escludono dal novero degli estimatori piú ferventi dell'opera consoliana, riconoscono unanimi nel libro, in questo libro, un classico: non sorprende perciò il suo inserimento in una collana ad hoc, né che qualcuno, come Sergio Pautasso, dichiari apertamente il piacere di rileggerlo o che qual-

coinvolgimento emozionale, né fingere un'ipocrita oggettività: memoria emotivamente condivisa per i protagonisti che ancora camminano per le strade, gesticolano odiano amano, continuano a resistere come possono, e s'incazzano in questo smemorato Occidente dove la supponenza della mondializzata economia di nuovo si autocelebra, in nome del mercato e del profitto, universale essenza dell'uomo contro l'uomo. E la sua spregiudicata ancilla - la politica - l'asseconda, insieme a Marx e a Voltaire, gettando l'utopia, come un nastro smagnetizzato, nelle discariche della storia.» (p. 35)

5. F. Di Legami, Vincenzo Consolo. La figura e l'opera, Marina di Patti (Messina): Pungitopo, 1990.

6. Nuove Effemeridi. rassegna trimestrale di cultura, 29, [Palermo: Guida] 1995.

7. A. SCUDERI, Scritture senza fine. Le metafore malinconiche di Vincenzo Consolo, Enna: Il Lunario, 1998.

8. G. Traina, Vincenzo Consolo, Fiesole (Firenze): Cadmo, 2001.

9. E. PAPA, «Ritratti critici di contemporanei: Vincenzo Consolo», Belfagor, LVIII 344, 2003, 179-198.

10. V. CONSOLO, Il sorriso dell'ignoto marinaio, "Oscar classici moderni. 193», Milano: Mondadori, 2004, p. XIV-XVII.

11. Ancora in corso di stampa gli atti del convegno Vincenzo Consolo. Éthique et écriture, tenuto alla Sorbonne Nouvelle venerdì 25 e sabato 26 ottobre 2002, con interventi di Guido Davico Bonino, Maria Pia De Paulis, Denis Ferraris, Giulio Ferroni, Rosalba Galvagno, Walter Geerts, Valeria Giannetti, Claude Imberty, Jean-Paul Manganaro, Antonino Recupero, Marie-France Renard, Cesare Segre. Sono invece usciti quelli del convegno siracusano: Enzo Papa (ed.), Per Vincenzo Consolo. Atti delle giornate di studio in onore di Vincenzo Consolo (Siracusa, 2-3 maggio 2003), San Cesario di Lecce: Manni, 2004, con contributi di Paolo Carile, «Testimonianza» (p. 11-13); Maria Rosa Cutrufelli, «Un severo, familiare maestro" (p. 17-22); Rosalba GALVAGNO, "Destino di una metamorfosi nel romanzo Nottetempo, casa per casa di Vincenzo Consolo» (p. 23-58); Massimo ONOFRI, «Nel magma italiano: considerazioni su Consolo scrittore politico e sperimentale» (p. 59-67); Sergio PAUTASSO, "Il piacere di rileggere $I l$ sorriso dell'ignoto marinaio, o dell'intelligenza narrativa» (p. 69-80); Carla RICCARDI, «Inganni e follie della storia: lo stile liricotragico della narrativa di Consolo» (p. 81-111); Giuseppe TraINA, «Rilettura di Retablo» (p. 113-132). Le relazioni presentate alle giornate di studio sivigliane, Vincenzo Consolo. Per $i$ suoi $70(+1)$ anni (Universidad de Sevilla, Facultad de Filología, 15-16 ottobre 2004), costituiscono il nucleo di questo numero di Quaderns d'Italià. 
che altro, come Massimo Onofri, ammetta Consolo in un canone resistente allo stesso variare delle mode critiche. ${ }^{12}$

D'altronde, il ruolo principe rappresentato dal Sorriso nel corpus consoliano è a piú riprese e in vari modi e gradazioni sottolineato dallo stesso autore: in interviste ${ }^{13}$ o anche in interventi sparsi, dalla postfazione all'edizione mondadoriana del ventennale ${ }^{14}$ alla lectio magistralis in occasione dell'investitura a doctor honoris causa dell'Università di Roma Tor Vergata (18 febbraio 2003).

Delineato tale scenario, arduo intervenire su quest'opera. Per l'occasione, quindi, con formula ciceroniana, mihi fines terminosque constituam e sottoporrò al dibattito critico un qualcosa di forse piú congeniale alla mia natura di manovale della filologia: un tentativo di tracciare una storia o, meno ambiziosamente, una cronaca del farsi del libro dalla sua "preistoria» in avanti, sistematizzando materiali sparsi, piú o meno noti, e aggiungendovi, con cautela, alcuni elementi nuovi. Una certa prudente reticenza è peraltro consigliabile, dettata com'è dallo svolgimento in atto di una ricerca, ormai quasi in dirittura d'arrivo, intesa proprio all'allestimento di una edizione critico-genetica del Sorriso. Insomma, ricorrendo alle categorie di avantesto, paratesto e testo, le mie intenzioni saranno piú perspicue e, ancor di piú, se si preciseranno le coordinate di una prospettiva ecdotica in cui nulla va «ricostruito», perché niente è stato "distrutto"; e nemmeno si tende a restituire in via ipotetica un archetipo smarrito e forse mai tangibilmente esistito, per definizione optimus e via via degradatosi nelle sue imperfette, corrotte riproduzioni, giacché l'opera, nella lezione licenziata dall'autore, è a nostra portata di mano. È una prospettiva di contro piú complessa e solo nominalmente, per cosí dire, capovolta: in essa, difatti, i testimoni recentiores, già dopo Giorgio Pasquali ammessi non deteriores, non sono però di necessità accettabili come senz'altro meliores — anzi meta raggiunta, immigliorabile e addirittura ottima dell' iter creativo e pertanto suggellati dal definitivo ne varietur dell'autore. Essi semmai presuppongono, trovano giustificazione fondante nei testimoni antiquiores, o piuttosto antiquissimi (dalla nota sparsa allo scartafaccio, ai prodotti delle successive fasi e decantazioni scrittorie), i quali al cospetto dei recentiores o ultimi, espressione dell'optima voluntas dell'autore, sarebbero certo da considerare tout court

12. Cfr. rispettivamente i saggi accolti in E. PAPA, (ed.), Per Vincenzo Consolo, op. cit., p. 69-80 e 59-67.

13. Dalle lontane Mario Fusco (ed.), "Questions à Vincenzo Consolo», La Quinzaine Littéraire, 321, 1980, 16-17; a Marino SINIBALDI (ed.), «La lingua ritrovata: Vincenzo Consolo", Leggere, 2, 1988, 8-15; dalla piú organica uscita in volume dal titolo guttusiano (è la didascalia di un quadro [olio su tela, cm.147,2 x 256,5] del 1940, finito l'anno prima e conservato alla Galleria Nazionale d'Arte Moderna di Roma), V. Consolo, Fuga dall'Etna. La Sicilia e Milano, la memoria e la storia, Roma: Donzelli, 1993, a quelle recentissime, l'una a cura di G. Traina, Vincenzo Consolo, op. cit., p. 123-138, o l'altra leggibile in internet, a cura di Dora MARRAFFA e Renato CORPACI, Italialibri, www.italialibri.net, 2001.

14. V. Consolo, Il sorriso dell'ignoto marinaio. Romanzo, con «Nota dell'autore, vent'anni dopo", "Scrittori italiani», Milano: Mondadori, 1997, p. 173-183; poi in ID., Di qua dal faro, Milano: Mondadori, 1999, p. 276-282, ed anche nell'ultima riproposta del Sorriso, ed. 2004, p. 167-175. 
destituiti di tutti i valori loro attribuibili dalla stemmatica classica, in quanto - pur prossimi al codice $x$ dell'opera - non si collocherebbero al di sotto di esso, non ne costituirebbero una fase cronologica piú bassa, inferiore, bensí soltanto e nient'altro che il piú alto, superiore e superato, perciò trascurabile, stadio magmatico embrionale. E tuttavia, per ciò stesso, tali reperti vanno sottoposti ad accurata recensio e collatio, e risultano necessari e imprescindibili per studiare il di-venire del testo dalla prima intelaiatura verso la tessitura rifinita, proprio perché nella genesi dell'opera rappresentano il caos primordiale, l'arché primigenia, non formata, l'impulso d'avvio e soprattutto la prova dei vari movimenti del testo fino al risolutivo colpo di timone dell'autore, insomma una sorta di illuminante pre-archetipo. ${ }^{15}$

\section{L'emerso}

Per comodità converrà sin dall'inizio tracciare la mappa delle edizioni a stampa [in grassetto l'ulteriore precisazione cronologica], anche perché sono quelle consultabili ed accessibili, e ad esse si rimanderà spesso:

1969 «Il sorriso dell'ignoto marinaio», Nuovi Argomenti, Nuova Serie, n. 15 [luglio-settembre]: edizione parziale, cap. I, senza Antefatto né Appendici I e II;

1975 Il sorriso dell'ignoto marinaio, Milano: Gaetano Manusé, edizione numerata con un'incisione firmata di Renato GUTTUSO: edizione parziale, cap. I, con Antefatto e Appendici I e II; e cap. II, L'albero delle quattro arance, senza Appendici I e II [autunno];

1976 Il sorriso dell'ignoto marinaio, Torino: Einaudi: editio princeps [finito di stampare 10 luglio, $1^{a}$ ed.; 18 settembre, $3^{\text {a }}$ ed.];

1987 Il sorriso dell'ignoto marinaio, intr. Cesare SEGRE, «Oscar oro. 9», Milano: Mondadori [marzo];

1992 Il sorriso dell'ignoto marinaio, «Nuovi Coralli. 464», Torino: Einaudi;

1995 Il sorriso dell'ignoto marinaio, ed. commentata a cura di Giovanni TESIO, intr. Cesare SEGRE, «Letteratura del Novecento", Milano: Elemond Scuola [dicembre];

1997 Il sorriso dell'ignoto marinaio. Romanzo, con «Nota dell'autore, vent'anni dopo», «Scrittori italiani», Milano: Mondadori [febbraio];

2002 Il sorriso dell'ignoto marinaio, "Oscar scrittori del Novecento", Milano: Mondadori [gennaio];

2004 Il sorriso dell'ignoto marinaio, "Oscar classici moderni. 193», Milano: Mondadori [marzo].

15. Solo qualche riferimento bibliografico ormai canonico: Louis HaY (ed.), Essais de critique génétique, Paris: Flammarion, 1979; Amos SEgALA (ed.), Littérature Latino-américaine et des Caraïbes du XX siècle. Théorie et pratique de l'édition critique, Roma: Bulzoni, 1988; Almuth GRÉSILLON, Éléments de critique génétique. Lire les manuscrits modernes, Paris: P.U.F., 1994; Giuseppe TAVANI, «Filologia e genetica», Cuadernos de Filología Italiana, 3 (1996): 63-90; Michel Contat \& Daniel Ferrer (edd.), Pourquoi la critique génétique? Méthodes, théories, Paris: CNRS Éditions, 1998. 
Non è agevole fissare date precise di avvio di una scrittura, neanche — si sa - nel caso di scrittori ancora produttivi con cui poter dialogare. Nel caso del nostro libro, ad ogni modo, tutto il movimento del testo - è ovvio - sarà iniziato verosimilmente tra l'a quo di La Ferita dell'aprile, il «mese piú crudele» di eliotiana memoria, cioè il $1963,{ }^{16}$ e la prima "orditura» licenziata dall'autore: quel Il sorriso dell'ignoto marinaio apparso su Nuovi Argomenti (luglio-settembre 1969), che corrisponde grosso modo al futuro cap. I del libro, ma non è ancora corredato né dell'Antefatto, né delle due Appendici documentarie a firma del protagonista, il barone Enrico Pirajno di Mandralisca. ${ }^{17}$

E un dato accertato, comunque, che le pagine appena ricordate, già dotate evidentemente, all'avviso dell'autore, di una loro autonomia e compiutezza narrativa, erano state in precedenza mandate, ma senza esito, alla rivista Paragone di Roberto Longhi e Anna Banti. A motivare l'invio è appunto il Trittico siciliano di Longhi, scritto in occasione della grande mostra del 1953 a Messina su Antonello e la pittura del ' 400 in Sicilia, ma il critico, in un incontro pubblico a Milano nel 1969, all'autore che chiedeva notizie del suo racconto cosí rispondeva severamente: «Sì, sì, mi ricordo benissimo. Non discuto il valore letterario, però questa storia del ritratto di Antonello che rappresenta un marinaio deve finire!». ${ }^{18}$ Rievocando l'episodio, Consolo cerca di giustificarlo cosí:

Longhi, nel suo saggio, polemizzava con la tradizione popolare che chiamava il ritratto del museo di Cefalù «dell'ignoto marinaio», sostenendo, giustamente, che Antonello, come gli altri pittori allora, non faceva quadri di genere, ma su commissione, e si faceva ben pagare. Un marinaio mai avrebbe potuto pagare Antonello. Quello effigiato lí era un ricco, un signore.

Lo sapevo, naturalmente, ma avevo voluto fargli «leggere» il quadro non in chiave scientifica, ma letteraria. ${ }^{19}$

Il testo veniva, allora, risolutamente spedito a Enzo Siciliano ed usciva finalmente su Nuovi Argomenti, la rivista di Alberto Carocci, Alberto Moravia e Pier Paolo Pasolini. La memoria personale dell'autore, corroborata dalla testimonianza di Caterina Pilenga, conosciuta subito dopo il trasferimento a Milano nel Capodanno del 1968, e da un certo punto in possesso di «ambo le chiavi | del cor» consoliano, ${ }^{20}$ questa doppia memoria fornisce altri dati di notevole interesse nella cronologia del farsi dell'opera.

16. Dal colophon si estraggono i seguenti dati piú precisi: «[...] impresso nel mese di settembre dell'anno 1963 [...] Il Tornasole — Pubblicazione periodica mensile - Registrazione Tribunale di Milano n. 6273 del 14-3-1963 [...]». Dell'opera si attende l'imminente versione spagnola a cura di Miguel Ángel Cuevas.

17. La pubblicazione — sia concessa l'indiscrezione - avrebbe fruttato all'autore un compenso di Lit. 16.000. In una lettera della direzione della rivista del 12 dicembre 1969, infatti, si conferma l'avvenuta pubblicazione (nel «numero testé pubblicato») e si comunica l'emissione di un assegno di tale importo.

18. V. Consolo, Fuga dall'Etna, op. cit., p. 37-38.

19. Ibid., p. 38.

20. Cosí viene presentata la futura moglie: «una delle cinque o sei persone che avevano letto» con entusiasmo la Ferita su segnalazione di Raffaele Crovi (Ibid., p. 35). Il quale è tra l'altro fra 
In primo luogo, riporta la chiusura del racconto, con quelle verosimili fattezze, a quell'anno 1968 e informa dell'avvenuta stesura, a quella data, e prima dell'arrivo a Milano nel gennaio 1968, anche del futuro cap. II L'albero delle quattro arance; inoltre, conferma che, dopo il fisico manifestarsi in Nuovi Argomenti, il progetto narrativo, di cui il racconto pubblicato è la prima concretizzazione, viene momentaneamente accantonato, anche se l'autore è nel frattempo preso dalla stesura del futuro cap. III Morti sacrata, che nessuno ha letto, tranne la moglie Caterina, e di cui alcuni sono a conoscenza (Corrado Stajano); infine, aggiunge che nel 1975 Consolo ottiene dalla RAI, nella cui sede milanese lavorava, ${ }^{21}$ un permesso di sei mesi, lascia Milano e torna in Sicilia dove collabora al giornale L'Ora di Vittorio Nisticò ${ }^{22}$ ed è raggiunto quell'estate da Caterina.

i pochi frequentati da Consolo, oltre al conterraneo Basilio Reale, sin dal tempo del primo soggiorno milanese (G. TraINA, Vincenzo Consolo, op. cit., p. 11): sono i tre anni della frequenza della Cattolica (1952-56), che saranno poi seguiti dal servizio militare a Roma, dalla laurea a Messina, dal praticantato notarile, dall'inizio del lavoro d'insegnante nel 1958 (E. PAPA, art. cit., p. 194).

21. A sottolineare i difficili rapporti di lavoro, l'azienda viene definita, una «fabbrica di armi» (V. Consolo, Fuga dall'Etna, op. cit., p. 34).

22. Vale la pena di riportare sull'esperienza giornalistica consoliana un brano dello stesso V. NisTicò, Accadeva in Sicilia. Gli anni ruggenti dell' "Ora» di Palermo, I, Palermo: Sellerio, 2001, p. 113-114: «[...] Vincenzo Consolo, sebbene vivesse ormai a Milano, da inquieto esule qual era, non perdeva occasione per tornare in Sicilia, dai suoi a Sant'Agata, e far sosta, potendo, anche al giornale. In fondo, tra i nostri scrittori era quello che sentivamo piú di casa, il piú famigliare. Amavamo di lui il garbo, la modestia, il senso di amicizia, gli accenni di sorridente ironia, non meno di quanto ci affascinassero i ricami della sua scrittura, la sua totale mediterraneità, quei fuochi improvvisi della sua passione letteraria e civile. Tra il ' 68 e il ' 69 pubblicammo una sua rubrica di annotazioni, "Fuori casa", un piccolo gioiello di giornalismo che diventa letteratura. || Nei primi mesi del '75 Consolo si trasferì per un po' di tempo a Palermo; glielo avevo chiesto perché ci desse una mano in vista delle importanti elezioni amministrative di giugno e di un evento che ci interessava direttamente: la candidatura di Leonardo Sciascia al consiglio comunale di Palermo. Era, la venuta di Consolo, un ritorno in redazione dopo l'esperienza di alcuni anni prima, quando si era trasferito da Sant'Agata per lavorare al giornale e impratichirsi del mestiere. Ma si era trattato di un'esperienza durata relativamente poco, interrotta dalla decisione di andarsene a Milano e dare, da allora in poi, la priorità assoluta alla letteratura; sarebbe stata lei la sua vita, il suo destino. || Tuttavia un desiderio di giornalismo, sebbene latente, rimase sempre vivo, e pronto a venir fuori quando si presentava l'occasione buona. Fu cosí in quei mesi del '75, quando facendo la spola tra la casa materna di Sant'Agata e la nostra redazione, si buttò con manifesta gioia in un intenso lavoro giornalistico. Partecipando dapprima con articoli e interviste alla campagna per il buon governo e la candidatura di Sciascia, poi nell'estate andando in giro col taccuino del cronista a seguire a Trapani il processo al «mostro di Marsala» (l'uomo che aveva fatto morire tre bimbe gettandole vive in un pozzo), o la vicenda del sequestro Corleo, il patriarca delle esattorie. In pieno agosto, si era persino spinto, e credo anche divertito, a fare un «viaggio» di osservazione tra gli uffici semideserti di Palermocapitale. Insomma, un bel bagno mediterraneo di umile giornalismo, mentre tra un servizio e l'altro trovava il luogo e il silenzio dove ripararsi per dare gli ultimi ritocchi a «Il sorriso dell'ignoto marinaio»: il capolavoro che da lí a qualche mese lo avrebbe consacrato tra gli eredi della grande letteratura che la Sicilia ha dato alla nazione. A dicembre ne pubblicammo in anteprima un capitolo: la festa in casa del barone Mandralisca.» 
Siamo dunque, estate del 1975, alla vigilia dell'edizione numerata in 150 esemplari con incisione firmata di Renato Guttuso per i tipi di Gaetano Manusé, edizione nel cui colophon è dichiarata la data dell' "autunno MCMLXXV». Manusé, da Valguarnera Caropepe di Sicilia, titolare prima di una bancarella poi di una libreria antiquaria a Milano, si era dichiarato interessato a pubblicare qualcosa di Consolo e, saputo dalla moglie Caterina, sollecitata in tal senso, dell'esistenza di un prosieguo del racconto già apparso sulla rivista moraviana, propone la pubblicazione per bibliofili del Sorriso. Basta ricordare che della composizione e tiratura si occuperà Martino Mardersteig della Stamperia Valdonega di Verona, erede della prestigiosa Officina Bodoni di Verona fondata dal padre Giovanni Hans, e che per l'occasione Leonardo Sciascia coinvolgerà Renato Guttuso il quale, rileggendo il ritratto di Antonello, appresterà un'incisione in cui viene rovesciata l'angolazione dell'immagine rispetto all'attante: il trequarti del misterioso personaggio non è rivolto a sinistra, ma a destra. ${ }^{23}$

Domenica 30 novembre 1975, la pagina culturale di Il Giorno di Milano pubblica un lungo articolo di Corrado Stajano, dal titolo redazionale molto allettante. ${ }^{24} \mathrm{Al}$ corrente delle alterne, combattute vicissitudini dello scriptorium di Consolo, conscio di quanto vi sta accadendo, Stajano fa una mossa a sorpresa: recensisce il libro appena uscito, ma ad un tempo, parlandone come della parte di un tutto imminente, sembra voler forzarne la definitiva confezione. Dopo aver presentato, difatti, le attività del libraio, cosí scrive:

Adesso Manusé ha esaudito il gran sogno della vita, è diventato editore e c’è la possibilità, dicono gli uomini di penna, che questo primo libro che ha stampato, $[\ldots]$ possa creare un nuovo caso letterario. Perché qui si sono incontrate due corde pazze siciliane, quella di Manusé e quella dello scrittore del libro, o meglio dei primi due capitoli del libro pubblicati in questo volume, che gli editori, quando il romanzo sarà finito, certo si contenderanno, perché «Il sorriso dell'ignoto marinaio» è un nuovo "Gattopardo», ma piú sottile, piú intenso del romanzo di Tomasi di Lampedusa, uno Sciascia poetico, di venosa lava sanguigna e insieme razionalmente freddo nei suoi teoremi dell'intelligenza. Uno scritto che arriva dentro l'impensata bottiglia di Manusé e che non ha nulla in comune con nessuno dei 17 mila libri che si pubblicano ogni anno in Italia.

Gli articoli pubblicati nel 1975 sono: «Un moderno Ulisse fra Scilla e Cariddi. Sfogliando il Gran libro di Stefano D’Arrigo" (22 febbraio); "L'avventurosa vita di Emilio Isgrò» (4 aprile); «Il malgoverno e l'impegno politico di Sciascia. Conversazione con Alberto Moravia» (30 maggio), "Il malgoverno e l'Università. Conversando con il Rettore dell'Università di Palermo, Giuseppe La Grutta» (13 giugno); vari servizi per il «Processo al "Mostro di Marsala"» (20, 21, 25, 30 giugno; 5, 11 luglio) e sul sequestro dell'esattore Luigi Corleo (18, 19 luglio); "A colloquio con il tenore Di Stefano» (14 luglio); «Tanta scienza e un po' di show" (26 luglio); "Che ne pensa Grassi, sovrintendente della Scala, del "caso Lanza Tomasi”?» (29 luglio); "In giro per gli uffici ad agosto» (9, 13 agosto); «Il giallo Majorana visto da Sciascia» (9 settembre).

23. L'incisione all'acquaforte viene eseguita a Palermo, in una stamperia vicina alla Galleria Arte al Borgo frequentata dallo scrittore di Racalmuto.

24. C. STAJANO, "Il sorriso dell'ignoto marinaio. Due siciliani pazzi per un libro "unico"», Il Giorno, Domenica 30 novembre 1975, 3. 
E piú avanti, in chiusa, fornisce anticipazioni sulla fabula e sprona, quasi rimbrotta l'autore:

Vincenzo Consolo, con tutte le sue contropoetiche, politicamente motivate, è troppo scrittore per rinunciare a scrivere, come avrebbe voluto. Gli è successa la sorte descritta da Roland Barthes ne "Il grado zero della scrittura»: «Partito per uccidere la letteratura, l'assassino si ritrova scrittore». [...] ora sta lavorando ai capitoli finali del romanzo, la rivoluzione contadina di Alcara Li Fusi, la repressione dello Stato italiano dopo la speranza portata da Garibaldi. Interdonato è il procuratore generale del processo contro i contadini, violenti contro la violenza. Mandralisca gli scrive una lunga memoria, i contadini cercano di narrare loro, la loro storia. Ci riusciranno?

«Il sorriso dell'ignoto marinaio» [...] è l'ultima difesa di uno scrittore che non voleva scrivere piú perché, quando il mondo s'incendia, la vita è meglio viverla che raccontarla.

C'è da pensare che, sotto la forte pressione morale-psicologica delle tre colonne di Stajano, Consolo raccogliesse il guanto della sfida che vi era insita e che, nello scorcio del 1975 e il primo semestre del 1976, con un lavoro che non si fa fatica ad immaginare, con il Leopardi da lui tanto amato, «matto e disperatissimo", stendesse e organizzasse il resto dell'opera: gli attuali capitoli IV-IX. Einaudi finisce, infatti, di stampare la prima edizione del libro quale sarà conosciuto dal vasto pubblico, l'editio princeps, il 10 luglio 1976 e ne farà circolare altre due stampe identiche, la terza licenziata il 18 settembre dello stesso anno.

\section{Tra emerso e sommerso}

Questi in buona sostanza i punti fermi del farsi del testo, i momenti fondanti della sua storia esterna. Se ne trae l'immediata idea di un progetto in crescendo, in progressione geometrica. ${ }^{25}$ Ma questi dati, relativi al merito e alle vicende dei soli testimoni a stampa, rappresentano solo l'emerso del testo e, in una prospettiva ecdotica critico-genetica, vanno naturalmente confrontati con quelli di quante altre fonti sia ancora possibile sottoporre a recensio e collatio. E qui, come anche per ogni altra opera di qualsivoglia altro scrittore, qualunque sforzo risulterebbe vano se l'autore volesse tutelare ad oltranza la legittima riservatezza della propria fucina, del proprio scriptorium. Il lavoro insomma

25. Forzando la suggestiva immagine del fondamentale saggio di Cesare SEGRE, «La costruzione a chiocciola nel Sorriso dell'ignoto marinaio di Vincenzo Consolo», in ID., Intrecci di voci. La polifonia nella letteratura del Novecento, Torino: Einaudi, 1991, p. 71-86 (trattasi dell' 'Introduzione» dell'edizione 1987 del Sorriso, p. V-XVIII, ripubblicata in quella del 1995, p. V-XIX), è come se tessere autonome (dal racconto iniziale, cap. I e II, all'integrazione del resto) si siano andate collocando a formare il mosaico dei gradini della scala tortile, ad imbuto dantesco, che — se si vuole accogliere l'interpretazione dei simboli di G. TRAINA, Vincenzo Consolo, op. cit., p. 61-70 — avrebbe consentito la discesa agli inferi e l'ascesa salvifica del protagonista. 
si bloccherebbe o potrebbe andare avanti solo con le carte di scrittori conservate in biblioteche, fondazioni, centri appositi (l'esempio piú noto, Pavia) o variamente e comunque riscattate, come per gli oltre 40 volumi già pubblicati della Collection Archives, ${ }^{26}$ il cui comitato scientifico è presieduto dal prestigioso romanista italiano Giuseppe Tavani. ${ }^{27}$

Nel caso del Sorriso, la generosa disponibilità dei coniugi Consolo, informati della necessità di queste esplorazioni per il mio studio, e in particolare l'amorevole scrupolosità di Caterina nel preservare materiali rivelatisi preziosi, hanno consentito di accumulare ingente informazione sulla scorta degli altri testimoni superstiti: tre bozze di stampa, di cui una eliminanda perché descripta, tre cartellette di dattiloscritti e un fascicolo dattiloscritto rilegato con l'opera intera; cinque manoscritti.

Ma prima, per completare il quadro dell'emerso, bisognerà rendere conto anche della contemporanea attività scrittoria del Nostro, in qualche misura dialogante con il progetto non ancora ben definito in quel lasso di tempo. La preistoria del Sorriso, quei tredici anni di lunga gestazione, grosso modo dal 1963 al 1976, sono affiancati da altre scritture.

Da una parte, le collaborazioni giornalistiche, tra cui spiccano: la rubrica Fuori casa, tenuta su L'Ora di Palermo; ${ }^{28}$ e vari reportages per Tempo illustrato. Ai fini dello studio del Sorriso sembrano importanti diversi di tali scritti. In primo luogo, il racconto Per un po' d'erba ai limiti del feudo, uscito prima su

26. La collana, diretta da Amos Segala e posta sotto il patrocinio dell'UNESCO, è affidata a un Consiglio di firmatari europei e latino-americani del Protocollo Archivos - ALLCA XX (Association Archives de la Littérature Latino-américaine, des Carä̈bes et Africaine du XXe siècle) e sottoposta alla valutazione di un Comitato scientifico internazionale. Le pubblicazioni seguono le indicazioni emerse dai seminari di Parigi (1984) e Oporto (1985), poi confluite nel volume di A. SEGALA (ed.), Littérature Latino-américaine et des Caraïbes du XXe siècle, op. cit.

27. Oltre all'art. cit., imprescindibili sono per equilibrio e dottrina: G. TAVANI, «Le Texte: son importance, son intangibilité»; «Teoría y metodología de la edición crítica», «Los textos del Siglo XX», "Metodología y práctica de la edición crítica de textos literarios contemporáneos»; "L'édition critique des auteurs contemporains: vérification méthodologique», tutti in A. SEgALA (ed.), Littérature Latino-américaine et des Carä̈bes du XXe siècle, op. cit., rispettivamente: p. 23-34, 35-51, 53-63, 65-84, 133-141. Cfr. inoltre: G. TAVANI, «L'edizione critico-genetica dei testi letterari: problemi e metodi», in Venezia e le lingue e letterature straniere. Atti del Convegno, Università di Venezia, 15-17 aprile 1989, Roma: Bulzoni, 1991, p. 323-331; «L'apporto dell'edizione di testi moderni alla pratica ecdotica, ovvero: l'apporto della pratica ecdotica all'edizione di testi moderni», in Anna FerRARI (ed.), Filologia classica e filologia romanza: esperienze ecdotiche a confronto. Atti del Convegno di Roma, 25-27 maggio 1995, Spoleto: Centro Italiano di Studi sull'Alto Medioevo, 1998, p. 545-554.

28. Cfr. l'elenco completo degli articoli firmati da Consolo per il giornale in V. Nisticò, Accadeva in Sicilia, op. cit. In particolare, la rubrica Fuori casa inizia il 7 dicembre 1968 e va avanti con cadenze irregolari per tutto il primo semestre del 1969 (11 gennaio, 24 febbraio, 10 marzo; 5, 24 e 25 maggio). Dello stesso anno sono: la recensione a Elio VitTORINI, Le città del mondo (27 settembre 1969) e un articolo sui rapporti tra mafia siciliana e americana (30 settembre 1969). 
$L^{\prime} O r a,{ }^{29}$ poi in un'autorevole silloge di narratori siciliani: ${ }^{30}$ un racconto strutturato come cronaca di una visita a Tusa alla famiglia di Carmine Battaglia, ucciso dalla mafia, in cui si innesta un breve brano documentario del 1860 sull'avversione dei nobili latifondisti al decreto garibaldino del 2 giugno 1860 lesivo dei propri privilegi. L'impianto rappresenterebbe quindi il primo, timido apparire, non piú di un accenno, di un modo costruttivo esemplato su modelli tedeschi, sul quale, per sua stessa affermazione, Consolo scommette con forza nel Sorriso ${ }^{31}$ e anche in seguito. ${ }^{32}$ Poi, su Tempo illustrato, un' inchiesta sui cavatori di pietra pomice delle Eolie affetti da silicosi, come quello dell' incipit del Sorriso, in pellegrinaggio al santuario di Tindari, ${ }^{33}$ e un'altra su Cefalù e quell'Aleister Crowley che apparirà molto dopo in Nottetempo, casa per casa (1992), e di cui si ha traccia in un quaderno ms del Sorriso che cosí contribuisce a datare. ${ }^{34}$ Infine, ancora su L'Ora, il resoconto dell'inaugurazione di una mostra di Guttuso, i cui appunti iniziali e primo svolgimento si trovano in un altro quaderno ms alla cui datazione ci si potrà cosí approssimare. ${ }^{35}$

Dall'altra parte, si annoverano le presentazioni di vari cataloghi di mostre, di cui due soprattutto rilevanti per la costituzione testuale del Sorriso: l'una di un'esposizione di Luciano Gussoni (1971), l'altra di un'esposizione di Michele Spadaro (1972), rilevanti in quanto i cataloghi sono latori di due lacerti rifusi rispettivamente nei capitoli VII e I. ${ }^{36}$

29. L'Ora, 16 aprile 1966. All'assassinio sono dedicati sul giornale, sempre in prima linea contro la mafia, articoli di Mauro DE MAURO (in seguito vittima della cosiddetta lupara bianca) e Mario Farinella (24, 25, 26, 28 marzo 1966) e di Felice Chilanti (9 aprile 1966).

30. Leonardo Sciascia \& Salvatore Guglielmino (edd.), Narratori di Sicilia, Milano: Mursia, 1967 , p. 428-434.

31. V. Consolo, Fuga dall'Etna, op. cit., p. 49.

32. Se si guarda solo alle opere limitrofe al Sorriso, il metodo sarà applicato, per le appendici erudite, a Lunaria, Torino: Einaudi, 1985, p. 71-85 (Milano: Mondadori, 1996, p. 93129) e, per gli inserti documentari, al racconto lungo "Ratumemi», in Le pietre di Pantalica, Milano: Mondadori, 1988, p. 47-74, altra storia di feudi del secondo dopoguerra, tematicamente piú affine a Per un po' d'erba...

33. "Così la pomice si mangia Lipari», Tempo illustrato, 17 ottobre 1970, di cui non si ha alcuna traccia nei mss. sottoposti a recensio. In $M s 2$ si riscontra invece la prima attestazione di «Una Sicilia trapiantata nella nebbia», che uscirà sempre su Tempo illustrato. L'articolo è conservato nel Fondo personale Consolo con l'annotazione di Caterina Consolo: «1970», senza indicazione del giorno e del mese, ma nel corpo si ravvisa un post quem: «ottobre».

34. Ms 2, ff. 1-5. Cfr. "C'era Mussolini e il diavolo si fermò a Cefalù», Tempo illustrato, 2 ottobre 1971 .

35. Ms 4, ff. $41^{\mathrm{v}}-33^{\mathrm{v}}$. Cfr. "Guttuso torna nella "sua" Milano", L'Ora, 18 ottobre 1974 . Sempre nell'ambito delle arti figurative, un altro articolo di alcuni mesi prima: «Bruno Caruso provoca Milano», L'Ora, 9 febbraio 1974.

36. V. Consolo, "Nottetempo, casa per casa», in Luciano Gussoni, Villa Reale di Monza, 10-30 novembre 1971; ID., "Marina a Tindari», in Michele Spadaro, Como, Galleria Giovio, 15-30 aprile 1972; poi anche in ID., Marina a Tindari, commento a cura di Sergio SPADARO, tiratura in cento esemplari numerati fuori commercio, Vercelli, Arti grafiche Cav. Piero De Marchi, 1972, p. 15-18. Quest'ultima presentazione è firmata e precisamente datata, com'è consuetudine dello scrittore: "Vincenzo Consolo \| (27 febbraio 1972)».

Nella fase preparatoria delle giornate di studio di Siviglia, ognuno in possesso e informato di un solo testimone, ci siamo scambiati i dati con il collega Miguel Ángel Cuevas. 


\section{Il sommerso}

Tornando ora ai testimoni manoscritti e dattiloscritti del Sorriso, non è questa la sede per proporne una descrizione esaustiva. Si cercherà invece di metterne in evidenza la portata facendo solo due esempi su versanti apparentemente diversi.

Intanto, sulla loro scorta, sarà possibile qualche correzione di tiro cronologica. Tra i quaderni mss, gli antiquiores, numerati appunto $M s$ e $M s$, contengono frammenti confluiti nella lezione di Nuovi Argomenti. Tra il 1969 e il 1975 si collocherebbero gli altri due, denominati $M s 3$ e $M s$ 4: sono latori, infatti, di lacerti non presenti nell'edizione 1969 e interpolati come due scatole cinesi in quella del 1975: l'uno, $M s$ 3, di un inciso avente per confini: «Lasciò la speronara [...] alla sua casa a Cefalù» (ff. 31-30v), l'altro, $M s 4$, di un ulteriore innesto nel tronco dell'inciso precedente: «Dietro questi pezzi [...] Caserta e di Versailles» (f. 18).

Questi stessi due quaderni Mss 3 e 4 sono inoltre legati dal ricordo, presente in entrambi, del primo incontro tra Leonardo Sciascia e Lucio Piccolo avvenuto in un giorno segnalato, il primo in cui grazie a una disposizione del Concilio Vaticano II si celebrava la messa in lingua italiana: domenica 7 marzo 1965. 37 L'appunto potrebbe essere trattato alla stregua di un indizio temporale e, per come e dove è tradito, una sorta di a quo / ad quem. ${ }^{38}$

Il riuso da parte dell'autore di $M s 4$, vergato capovolto, assicura poi la trasmissione dell'articolo giornalistico su Guttuso già ricordato e da datare perciò ante il 18 ottobre 1974.

Se, infine, contestualmente ai dati appena forniti, consideriamo che $M s 3$ tramanda varie stesure di Morti sacrata (futuro cap. III), le prime prove di Val Dèmone (futuro cap. IV), un appunto che rinvia a Il Vespro (futuro cap. V) e che Ms 4 tramanda brani di Val Dèmone e la Lettera di Enrico Pirajno all'avvocato Giovanni Interdonato (futuro cap. VI), si potrebbe inferire che, se non proprio intorno al 1965 (incontro Sciascia-Piccolo), già alla data del 1974 (articolo sulla mostra di Guttuso) o tutt'al piú, in ultima istanza, nel 1975 prima dell'edizione Manusé, il Sorriso fosse per buona parte, quasi per intero in movimento. Allo stato attuale, mancherebbero attestazioni mss databili solo dei capitoli VII, VIII, IX.

37. Cfr. $M s 3$, ff. $17^{\mathrm{v}}$ e $20 ; M s 4$, f. guardia $1^{\mathrm{v}}$.

38. L'appunto sarà sviluppato in Le pietre di Pantalica, op. cit., p. 142 e ricordato in Fuga dall'Etna, op. cit., p. 23-24, dove viene ulteriormente esteso (testo in corsivo nostro): "Al congedo, sulla porta, Piccolo solennemente disse allo scrittore, indicando con la mano su per le colline: "Sciascia, la invito a scrivere di queste nostre terre, di questi paesi medievali". "C’è qui Consolo", rispose Sciascia. "Consolo è ancora giovinetto", replicò Piccolo sarcasticamente (avevo trentatrè anni!). Ma io presi quella frase come impegno verso Sciascia e come una sfida verso il barone». L'interesse per il poeta aveva già dato frutto in un'intera pagina del giornale di Nisticò con un articolo: "Il barone magico: Lucio Piccolo", L'Ora, 17 febbraio 1967, accompagnato da quattro canti inediti. Si noti che "Il barone magico» è il titolo scelto da Consolo per il trittico che costituisce la penultima parte della sezione Persone, seconda e centrale di Le pietre di Pantalica, op. cit., p. 133-135, 136-144, 145-149. 
Se andiamo ora, secondo esempio, alle tre cartellette di dattiloscritti, se ne potrà ricavare informazione sia dai fascicoli contenuti, sia anche dai bifogli di cartoncino colorato (rosa) che li raccolgono e conservano. Ed è informazione di peso circa il crescere del progetto di scrittura e la graduale definizione dell'architettura dell'opera. Solo qualche breve accenno.

Si confronti ad es. la copertina della cartelletta denominata $D s 1$, contenente prime stesure dei capp. I-VI, con annotazioni a mano di Caterina Consolo, con varie modifiche di titolo, con quella della cartelletta designata $D s 3$, contenente tutta l'opera tranne il cap. VI (Lettera...), sulla quale appare già lo schema definitivo autografo con le date relative alla scansione del tempo interno dell'opera, in corrispondenza dei singoli capitoli: un'articolazione in tre parti (la prima: cap. I + App. I e II, cap. II + App. I e II; la seconda: capp. IIIV; la terza: capp. VI-IX) + Appendici finali, numerate «10)» e intitolate inizialmente «10) La fucilazione» e poi poste sotto l'epigrafe generica «10) Appendici»; e ancora qualche titubanza sulla collocazione di Morti sacrata (il capitolo prima segue «3) Val Dèmone» ed è quindi numerato «4)», ma poi entrambe le numerazioni vengono emendate ed invertite).

Ancora piú illuminante il fascicoletto numerato Ds 1.1, intitolato polisemicamente Carte per gioco e con l'eloquentissimo sottotitolo "(Racconti e cose da raccontare fin dal tempo di Garibaldi)", il quale sembra in tutto e per tutto lo schema strutturale di un'opera non nata, o piuttosto la crisalide che si trasformerà nella futura farfalla: ${ }^{39}$ le Carte sono articolate in tre tempi: «narrativo» (e sarebbe il Sorriso del 1969, quello di Nuovi Argomenti, preceduto però da un "Antefatto" scritto ex novo e seguito da un'appendice documentaria (Lettera di Enrico Pirajno barone di Mandralisca al barone Andrea Bivona), ${ }^{40}$ "storico» (con riportati brani documentari storici sulla strage di Alcara e un bollettino di guerra), "magico o poetico», dedicato a Lucio Piccolo, brano che con qualche variante vedrà la luce molto tempo dopo nelle Pietre di Pantalica. ${ }^{41}$

È evidente, e non può non sorprendere, come in tempi insospettati ed alti nella cronologia del Sorriso, fossero già tutti presenti i principali semi, gli elementi lievitati nel futuro libro: l'invenzione diegetica, l'analitico storico d'influenza tedesca, il poetico; ci fossero i personaggi e i fatti: insomma, come scrive Enrico Pirajno di Mandralisca, per un momento alter ego dell'autore, «il timbro e il tono, e le parole» (Sorriso, ed. 2004, p. 119). Sembra pure chia-

39. Il titolo è allusivo: nugae, carte da gioco (tre come i tempi), cartelle dss "per giocare», e verosimilmente anche nel senso traslato del jouer, del play, «da eseguire, interpretare, rappresentare». Ancor di piú il sottotitolo, con l'accenno al già raccontato (la propria pièce iniziale) e alle cose o fatti otto-novecenteschi ancora in cerca d'autore, un autore che sappia come raccontarli, e in quale chiave: diversa dalla canonica, allora, da quella suggerita dagli auctores?, non alla Verga, Pirandello, Tomasi, Sciascia?

40. Sarebbe la prima attestazione della futura «Appendice prima» del cap. I.

41. È il primo dei tre capitoletti riuniti — come già detto - sotto il titolo "Il barone magico» nella sezione Persone di Le pietre di Pantalica, op. cit., p. 133-135. 
ro come fosse già maturata la scelta del «romanzo storico-metaforico» ${ }^{42}$ con un occhio rivolto al Manzoni, ma superandone il paternalismo espressivo grazie all'insegnamento di Verga, ${ }^{43}$ e l'altro ai tedeschi del Gruppo 47, gli «analitici» Hans Magnus Enzensberger, Alexander Kluge ed altri, di cui aveva dovuto leggere pagine sul Menabò vittoriniano $(9,1966)$ e nelle traduzioni dei primi anni Settanta, ${ }^{44}$ e che lo riportavano forse al Manzoni che ritratta e, ormai spinto alla negazione dei suoi stessi precetti poetici, è capace solo di redigere la Storia della colonna infame $e^{45}$ che a tutti i costi vuol pubblicare in solido con I promessi sposi (1842). ${ }^{46}$

Scorgiamo già all'orizzonte, insomma, il Sorriso quale è arrivato a noi, e nella chiave e forma, scelte dall'autore, di "romanzo ideologico», cioè di romanzo «critico», di una ideologia che consiste «nell'opporsi al potere, qualsiasi potere, nel combattere con l'arma della scrittura, che è come la fionda di David, o meglio la lancia di Don Chisciotte, le ingiustizie, le sopraffazioni, le violenze, i mali e gli orrori del nostro tempo.» ${ }^{47}$

42. V. Consolo, Fuga dall'Etna, op. cit., p. 70; all'insegna della convinzione piú volte manifestata, ed esplicitata dall'esergo di questo stesso libro-intervista (p. 1), che: «Il solo coerente sistema di segni da cui può essere colta la storia come realtà materiale sembra essere la letteratura (H. M. ENZENSBERGER, Letteratura come storiografia)».

43. C. RiCCARDI, «Inganni e follie della storia », in E. PAPA (ed.), Per Vincenzo Consolo, op. cit., p. 91.

44. Ibid., p. 82 e p. 109, n. 3. E, prima, cfr. V. Consolo, Fuga dall'Etna, op. cit., p. 49.

45. Nell'a parte, quasi alla fine del cap. VII del Sorriso, viene alla fine omesso un brano dell'Introduzione della Storia manzoniana, che viene bensí riportato nella fonte di quel passo (in corsivo nostro il lacerto tradito da Luciano Gussoni, op. cit. e poi espunto): "Che vengano, vengano ad orde sferraglianti, con squilli lame della notte, perché il silenzio, la pausa ti morde. || Chi sparse quella peste? Nessuno. Nessuno con cuore d'uomo accese queste micce. "...La rabbia resa spietata da una lunga paura, e diventata odio e puntiglio contro gli sventurati che cercavan di sfuggirle di mano; o il timor di mancare a un'aspettativa generale...; il timor fors'anche di gravi pubblici mali che ne potessero avvenire». Ma già è tardi. Già sono state issate le colonne dell'infamia. || Ma tu aspetta, fa' piano. [...]» (Sorriso, ed. 2004, p. 130).

46. Un'incisiva descrizione della macerante riflessione manzoniana viene proposta da Giovanni AlberTocCHI, Alessandro Manzoni, Madrid: Síntesis, 2003, p. 106-116.

47. In questi termini viene esplicitata la definizione in V. CONSOLO, Fuga dall'Etna, op. cit., p. 70. Dalla facile accusa di ideologismo mette al riparo la pregnante valutazione di M. ONOFRI, «Nel magma italiano", in E. PAPA (ed.), Per Vincenzo Consolo, op. cit., p. 60: "Consolo, ecco il punto, è un miracoloso scrittore politico: laddove il miracolo sta nel fatto che la politica gli si eserciti sulla pagina per via di un'oltranza di stile.» 